\title{
Candida lusitaniae - a case report of an intraperitoneal infection
}

\author{
Sara Wawrysiuk, Tomasz Rechberger, Konrad Futyma, Paweł Miotła \\ $2^{\text {nd }}$ Department of Gynaecology, Medical University of Lublin, Lublin, Poland
}

\begin{abstract}
Candida lusitaniae is a rare opportunistic yeast which is known for its resistance to amphotericin B (AmB). It is responsible for about $19.3 \%$ of all infections caused by non-Candida albicans species, and for about $1.7 \%$ of all cases of genitourinary candidiasis brought about by the entire spectrum of Candida species. Most commonly it occurs in patients with hematologic malignancies, especially when a patient is receiving chemotherapy. Candida lusitaniae infection usually presents with fungemia; however, only $7.3 \%$ of all patients will develop peritonitis.

This case study describes an immunocompetent female patient with an intraperitoneal infection caused by $C$. lusitaniae after laparoscopic hydrosalpinx surgery. The patient was treated with fluconazole according to susceptibility testing. Fluconazole was implemented both orally and by transvaginal injection into the space after the evacuated pseudocyst. Conservative treatment resulted in a temporary improvement of the patient's condition and a reduction of the pseudocyst.

Candida lusitaniae is very similar to other Candida species in generating systemic and local infections mainly in compromised patients. It is also unique in its ability to develop resistance to AmB. Proper identification and quick implementation of selective therapy with the right anti-fungal drug are crucial for successfully treating infected patients. Surgery should always be considered as a final treatment option.
\end{abstract}

Key words: Candida lusitaniae, fluconazole, amphotericin B, intraperitoneal infection.

\section{Introduction}

Candida lusitaniae is a rare opportunistic yeast which is known for its resistance to amphotericin B $(A m B)$. The prevalence of $A m B$ resistance varies between 21 and 60\%, depending on the study [1]. Candida lusitaniae isolates not only show resistance to AmB, but some can also develop it in vivo during the treatment [2]. Reversible switching from resistant to sensitive AmB strains occurs as well, but at a much lower rate [3].

Candida lusitaniae is responsible for about $19.3 \%$ of all infections caused by non-Candida albicans species [4] and for about $1.7 \%$ of all cases of genitourinary candidiasis brought about by the entire spectrum of Candida species [5]. It occurs most commonly in patients with hematologic malignancies [6], especially when a patient is receiving chemotherapy. Other risk factors include neutropenia, long-term corticosteroid therapy, receipt of broad-spectrum antibiotics, bone marrow transplant, central venous catheter and dialysis [7].

Candida lusitaniae infection was considered fatal before the era of fluconazole. Nowadays, death among patients with $C$. lusitaniae is not very usual and does not exceed $5 \%$ of all cases [1]. The most common type of $C$. lusitaniae infection is fungemia. Indeed, in the study conducted by Hawkins et al. reviewing 55 patients with C. lusitaniae infection, $80 \%$ showed fungemia, yet only $7.3 \%$ of all patients presented peritonitis. The most common symptom of $C$. lusitaniae infection is fever [7].

This article describes a case of a patient with intraperitoneal $C$. lusitaniae infection after hydrosalpinx removal.

\section{Case presentation}

A 46-year-old female patient was admitted to our clinic for the surgical treatment of hydrosalpinx. The patient had a history of laparoscopic supracervical hysterectomy with the right fallopian tube due to uterine fibroids ten months prior to admission. On admission, she complained of abdominal pain. She had no fever and no other symptoms. Laboratory results showed: white blood cells $9.03 \mathrm{~K} / \mu \mathrm{l}$; red blood cells $4.28 \mathrm{M} / \mu \mathrm{l}$; hemoglobin $13.5 \mathrm{~g} / \mathrm{dl}$; platelets $209 \mathrm{~K} / \mu \mathrm{l}$; C-reactive protein $3.33 \mathrm{mg} / \mathrm{l}$; cancer antigen $1259.8 \mathrm{U} / \mathrm{ml}$. Vaginal ultrasound showed an enlarged fallopian tube with a fluid space (Fig. 1). Based on pelvic examination, laboratory results and ultrasound imaging, she was qualified for a laparoscopic hydrosalpinx removal. During laparoscopy, adhesions between the left fallopian tube, abdominal wall and cervical stump were released. Furthermore, an enlarged left fallopian tube with a diameter of about $4 \mathrm{~cm}$ and a brown, serum-like fluid reservoir next to the 
left ovary were exposed. After the evacuation of the fluid, the left fallopian tube was removed. The result of a histopathological examination confirmed a hydrosalpinx. The patient was discharged after three days, in a good condition.

Three months later, the patient was referred to our clinic again due to a very strong pelvic pain. Ultrasound examination revealed a multilocular, fluid reservoir $(77 \times 72 \mathrm{~mm})$ next to the cervical stump (Fig. 2). Transvaginal aspiration of $150 \mathrm{ml}$ of clear fluid was performed under ultrasound guidance. Subsequently, $300 \mathrm{mg} / 14 \mathrm{ml}$ of doxycycline was injected into the space after aspiration. The patient was discharged the same day, in a good condition.

At this time, a fluid sample was taken for culture, and Candida lusitaniae was isolated. The patient then had another transvaginal aspiration of a pseudocyst because of its recurrence (Fig. 3) performed in our clinic. A solution of $20 \mathrm{mg} / 10 \mathrm{ml}$ fluconazole was injected after aspiration of the pseudocyst's fluid. The patient was started on doxycycline (100 mg twice daily, orally), clarithromycin (250 mg twice daily, orally) and fluconazole (150 mg once daily, orally) and discharged home.

Currently, the patient is being referred to our clinic again due to a bilateral recurrence of pseudocysts. Because of the failure of conservative treatment, she is going to have pseudocysts removed surgically.

\section{Discussion}

Hydrosalpinx is a fluid-filled fallopian tube which usually gives no symptoms, although sometimes it can lead to pelvic pain, pelvic pressure or dyspareunia. The most common causes of hydrosalpinx are pelvic inflammatory disease, previous abdominal surgery, endometriosis, ectopic pregnancy or a congenital malformation [8]. The treatment may be difficult especially when a patient is trying to conceive. In perimenopausal women, the best treatment option is laparoscopic removal of the hydrosalpinx because it is associated with less bleeding and faster recovery.

Intra-abdominal infections are a major cause of morbidity in patients undergoing abdominal surgery. When it comes to Candida infection after abdominal surgery, it is mainly represented by, but not limited to, peritonitis or intra-abdominal abscesses [9]. Candida albicans is the most common fungal pathogen, but the prevalence of non-C. albicans is also not trivial. In the study conducted by Rodolico et al., 66 cases of intra-abdominal infections with Candida spp. were identified during a 5-year study period. Moreover, C. albicans was isolated in 35 patients, and non-C. albicans spp. (C. tropicalis 19\%; C. parapsilosis 16\%; C. glabrata 8\%; C. lusitaniae 7.5\%) in 31 patients. The prevalence of $C$. albicans and non-C. albicans species was comparable in this study, with non-C. albicans spp. being related mainly to critically ill surgical patients [10].

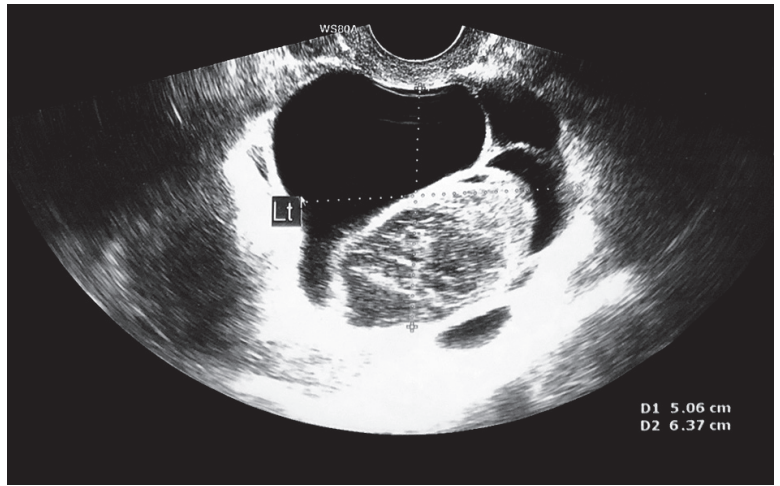

Fig. 1. Enlarged fallopian tube with a multilocular fluid space

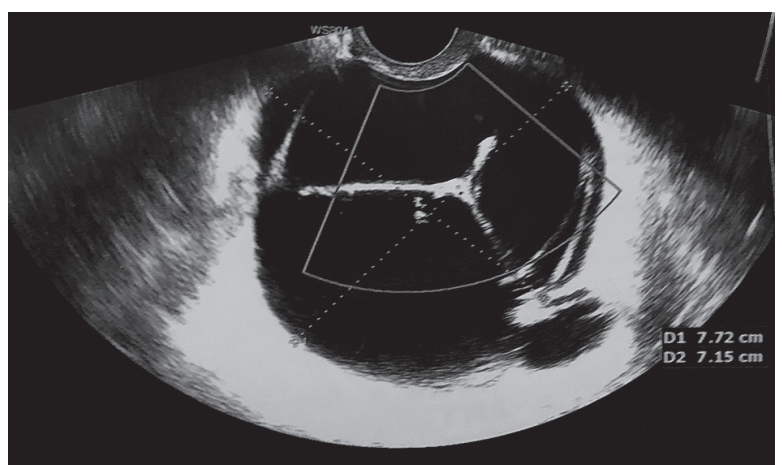

Fig. 2. Multilocular fluid reservoir next to the cervical stump three months after surgery

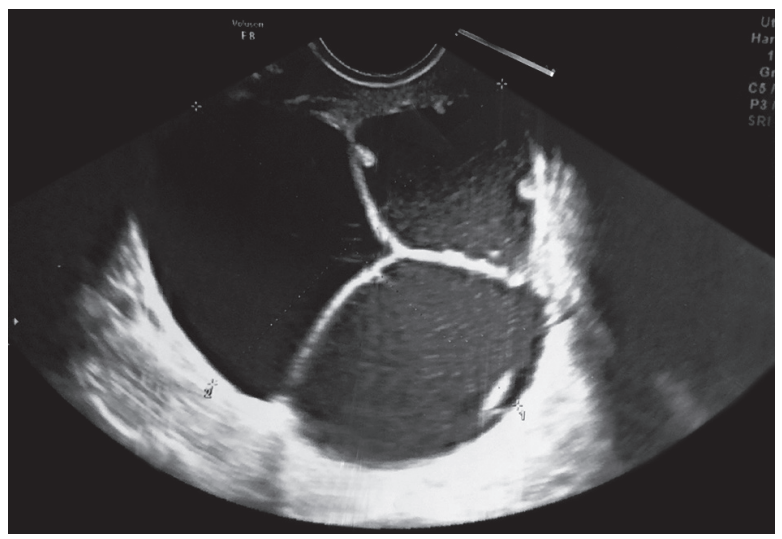

Fig. 3. Multilocular fluid reservoir after transvaginal aspiration and antibiotic treatment

Our case involved a very rare opportunistic yeast, Candida lusitaniae [4], which infected a healthy patient after a simple surgical procedure conducted in a sterile environment. The patient was first treated with broad-spectrum antibiotics due to the fact that bacteria usually cause an infection. After the culture result and according to the susceptibility testing, the patient was then switched to an anti-fungal drug. In this case, fluconazole administration resulted in temporary improvement of her condition and a reduction of the pseudocyst. Due to the failure of conservative 
treatment and recurrence of pseudocysts, the patient has to undergo surgery, which should be considered as a final treatment option.

The presented case study illustrates the importance of taking cultures, as these enable isolation, identification, and speciation of the pathogen. All isolates of C. lusitaniae should also undergo susceptibility testing. In addition, when the isolate is found to be susceptible to AmB and the patient is started on the treatment, it might be necessary to test the susceptibility of C. lusitaniae again during the treatment, especially if the patient's condition is not improving. This is due to the abovementioned ability of $C$. lusitaniae to develop resistance to $A m B$ during treatment.

\section{Conclusions}

Candida lusitaniae is very similar to other Candida species in causing systemic and local infections, mainly in compromised patients, but it is also unique in its ability to develop resistance to AmB. Proper identification and quick implementation of selective therapy with a dedicated anti-fungal drug are crucial for the successful conservative treatment of an infected patient. Surgery should always be considered as a final treatment option.

\section{Disclosure}

The authors report no conflict of interests.

\section{References}

1. Hawkins JL, Baddour LM. Candida lusitaniae infections in the era of fluconazole availability. Clin Infect Dis 2003; 36: e14-e18.

2. Cantón E, Pemán J, Gobernado M, et al. Patterns of Amphotericin B Killing Kinetics against Seven Candida Species. Antimicrob Agents Chemother 2004; 48: 2477-2482.

3. Yoon SA, Vazquez JA, Steffan PE, et al. High-frequency, in vitro reversible switching of candida lusitaniae clinical isolates from amphotericin B susceptibility to resistance. Antimicrob Agents Chemother 1999; 43: 836-845.

4. Jung DS, Farmakiotis D, Jiang $Y$, et al. Uncommon Candida Species Fungemia among Cancer Patients, Houston, Texas, USA. Emerg Infect Dis 2015; 21: 1942-1950

5. Obisesan OJ, Olowe OA, Taiwo SS. Phenotypic Detection of Genitourinary Candidiasis among Sexually Transmitted Disease Clinic Attendees in Ladoke Akintola University Teaching Hospital, Osogbo, Nigeria. J Environ Public Health 2015; 2015: 401340.

6. Blinkhorn JR, Adelstein D, Spagnuolo PJ. Emergence of a new opportunistic pathogen, Candida lusitaniae. J Clin Microbiol 1989; 27: 236-240.

7. Minari A, Hachem R, Raad I. Candida lusitaniae: a cause of breakthrough fungemia in cancer patients. Clin Infect Dis 2001; 32: 186-190.

8. John PR, Pasley AM. Torsion of Hydrosalpinx with Concurrent Acute Cholecystitis: Case Report and Review of Literature. Case Rep Surg 2016; 2016: 5424092.

9. Bassetti M, Marchetti M, Chakrabarti A, et al. A research agenda on the management of intra-abdominal candidiasis: results from a consensus of multinational experts. Intensive Care Med 2013; 39: 2092-2106.

10. Rodolico V, Di Carlo P, Gulotta G, et al. Intra-abdominal Candida spp. infection in acute abdomen in a quality assurance (QA)-certified academic setting. J Clin Pathol 2017; 70: 579-583. 